tung", so der Leiter des Instituts für Internet-Sicherheit, Professor Norbert Pohlmann.

Die IKS-Plattform ist unter iks.internet-sicherheit.de erreichbar.

\section{Sicherheit beim Online-Banking: Bürger zwischen Angst und Sorglosigkeit}

Immer mehr Bürger möchten ihre Bankgeschäfte mobil und bequem zu jeder Zeit erledigen. Aktuell nutzen 70 Prozent der regelmäßigen Internetnutzer häufig den Computer für ihre Bankgeschäfte. Über 95 Prozent von ihnen legen dabei großen Wert auf Sicherheit und Datenschutz. Dafür etwas tun oder bezahlen wollen aber immer weniger Menschen: Aktuell erwarten 57 Prozent der Internetnutzer sichere Transaktionen kostenlos von ihrer Bank. Dies sind die zentralen Ergebnisse der fünften Auflage der Studie „Online-Banking - Mit Sicherheit!", am 31.01.2013 herausgegeben von der Initiative D21 e.V. und der Fiducia IT AG und durchgeführt von TNS Infratest.

\section{Sicherheit und Datenschutz bewegen die Menschen}

Für die Bürger zählen beim Online-Banking am meisten Sicherheit und Datenschutz, ein Ergebnis, dass sich konstant durch die fünfjährige Historie der Studie zieht. „Information und Aufklärung zum Thema Sicherheit und Datenschutz im Netz sind zentrale Aufgaben der Initiative D21. Die geäußerten Befürchtungen und Ansprüche an die Sicherheit bei gleichzeitiger Sorglosigkeit und mangelnder Zahlungsbereitschaft sind ein Signal für uns, in diesem Gebiet noch stärker aktiv zu werden", so Robert A. Wieland, Vizepräsident der Initiative D21 und Geschäftsführer der TNS Infratest. Er stellte die Ergebnisse der Studie am 31. Januar 2013 in München der Öffentlichkeit vor.

Ebenfalls seit Beginn der Untersuchung zu verzeichnen ist die Angst, beim Online-Banking betrogen zu werden. Rund ein Viertel der Internetnutzer in Deutschland hält die Angst vor Betrug aktuell davon ab, Online-Bankgeschäfte zu tätigen. Diese Ängste sind besonders stark bei Menschen ausgeprägt, die Online-Banking nicht nutzen (72 Prozent). Anders die Online-Banking-Nutzer: 80 Prozent dieser Gruppe fühlen sich sicher.

\section{Zunehmende Nachlässigkeit der Nutzer}

Über die Jahre konstant ist auch der Trend, dass immer weniger Onliner trotz ihrer Ängste bereit sind, für Sicherheit bei Transaktionen im Internet zu zahlen. Inzwischen sind 57 Prozent der Befragten überzeugt, dass sicheres Online-Banking ein kostenloses Angebot ihrer Bank sein muss.
Parallel sichern immer weniger Menschen ihren Computer. "Nur noch 75 Prozent der Onliner schützen ihren PC mit speziellen Programmen, um die Sicherheit beim Online-Banking zu erhöhen. 2008 waren es immerhin 84 Prozent ", berichtet Wieland. Ein Grund für diese Sorglosigkeit könnte sein, dass bislang nur wenige Menschen, nämlich zwei Prozent der Befragten, durch eine Schadsoftware finanziell geschädigt wurden. Bei über der Hälfte der Geschädigten lag der Schaden unter 200 Euro.

Positiver sieht die Sicherheitsbilanz beim Online-Banking aus. Hier setzen sich die aktuell sichersten Techniken 2012 klar an die Spitze: 31 Prozent der Nutzer verwenden mobile TAN und 28 Prozent Sm@rtTAN plus. Bei den Volksbanken und Raiffeisenbanken im Geschäftsgebiet der Fiducia dürfte die Bilanz noch wesentlich positiver ausfallen. Hier nutzen inzwischen 2,4 Millionen Bankkunden das Sm@rt-TAN-plus-Verfahren und 1,7 Millionen die mobileTAN. Mit ein Grund für diesen hohen Sicherheitsstandard dürfte sein, dass die Fiducia bereits 2006 ihren Kunden mit der mobileTAN eines der sichersten Verfahren zur Verfügung stellte.

\section{Banking online und mobil}

Wie schon in den beiden Vorjahren, so ist auch 2012 für Internetnutzer der PC mit 70 Prozent der meist genutzte Weg zur Bank. Dennoch besuchen 44 Prozent der Onliner häufig eine Geschäftsstelle, um mit ihrem Berater zu sprechen. Wer ein Smartphone oder Tablet besitzt, nutzt immer häufiger auch diese Geräte für die Abwicklung von Bankgeschäften.

Aktuell verwenden 26 Prozent der Smartphone-Besitzer häufig das Smartphone und sogar 37 Prozent der Tablet-Besitzer häufig das Tablet für Bankgeschäfte. Der Erfolg und die Beliebtheit der von der Fiducia entwickelten Banking-App „vr.de" belegt den Trend zum einfachen und mobilen Banking. Für Anfang Februar 2013 wird der ein Millionste Download erwartet. 2013 ergänze außerdem ein Personal Finance Management das Angebot für den Bankkunden. Diese Lösung bedient ebenfalls den Wunsch des Kunden nach mehr Komfort beim Online-Banking, lässt sich damit doch ganz einfach das private Budget planen.

Wenig Beachtung bei den Onlinern finden dagegen die SocialMedia-Angebote der Banken. So erwarten aktuell nur zehn Prozent der Befragten, Angebote ihrer Bank bei Facebook zu finden. Mobile Payment ist dagegen ein Service, der noch auf seinen Durchbruch wartet. Fünf Prozent der Onliner nutzen aktuell entsprechende Angebote, acht Prozent planen, sie zu nutzen. 72 Prozent der MobilePayment-Nutzer und Nutzungsplaner nennen Banken und Sparkassen als Anbieter ihres Vertrauens.

Die Studie „Online-Banking - Mit Sicherheit!" steht unter www. initiatived21.de/publikationen und www.fiducia.de kostenlos zum Download zur Verfügung. 